\title{
Comparative Clouds
}

Kyle Flaherty, Rebecca Hack, Alina Pacheco ${ }^{1}$

Flaherty, K; Hack, R; Pacheco, A. Comparative Clouds. Tecnología en Marcha. Vol. 33, especial Movilidad estudiantil. Pág 155-165.

doi) https://doi.org/10.18845/tm.v33i7.5490 


\begin{abstract}
With the cloud industry still in its infancy, it is imperative to complete a thorough analysis of the major competitors in this market. The research covered in this paper provides a comparative analysis of the main competitors in the cloud industry: Amazon Web Services, Google Cloud, and Microsoft Azure. The focus of this analysis will be structured around usability, performance and cost. While the other competitors have their niche markets, the best overall cloud provider was Amazon WebServices.
\end{abstract}

\title{
Introduction
}

From the inception of the cloud computing industry multiple companies have come forth to supply the growing demand. The earliest suppliers, such as Ama- zon Web Services (AWS), were able to take advantage of the industry while there were few competitors. This made it difficult for later providers such asGoogle Cloud (GC) and Microsoft Azure as they attempted to provide offerings that could compete against the set industry standard. With the growing number of companiestha tareenteringthisindustry,forcompanies and individuals that use the cloud it is important that they evaluate the current industry offerings. By doing so, they can determine which cloud services will best match theirneeds.

The purpose of this paper is to evaluate the three most popular clouds, AWS, GC, and Azure, against three metrics to determine which is the bestoverall cloud service provider in the cloud computing industry. For the scope of this paper, the Infrastructure as a Service (laaS) segment of the cloud computingindustry will be the focus. The first metric evaluated is usability whi chwillcovertheprovider'savailablecloudservices, its number of data centers, and customer reviews on their strengths and weaknesses. The second metric is performance which will include an analysis of both hardware and software offerings that are available by the different laaS providers. The last metric is a cost analysis which will evaluate the costs for various servicesavailablethroughthelaaSproviders.

In the section to follow, we will provide a brief analysis of the current Cloud laaS market. The three sections after that will cover the usability,performance, and then cost analysis for the three cloudproviders we are analyzing. Finally, we will determine the best overall cloud provider based on the analysis from the previoussections.

\section{Market Research}

The recent emergence of the cloud industry has had a dramatic impact on the strategies of many businesses as they switch from using data centers to storing and managing their information on the cloud. In 2017, the marketvalueofthecloudcomputingindustrywaslisted at over \$18.5 billion after growing by 37.5\% from 2016 and similar growth is expected in the years to come [1]. This industry is split into three segments: Infrastructure as a Service (laaS), Platform as a Service (PaaS), and Software as a Service (SaaS). This paper focuses on laaS which accounts for $24.3 \%$ of the market value which equates to $\$ 4$ billion [2]. While there are numerous cloud providers in this industry, the market is mostly dominated by AWS (41.5\%market share) and Azure (29.4\% market share) [2]. Each of the other competitors, including GC, hold 3\% of themarket or less, but all theses small competitors combined account for for $29.2 \%$ of the market which is a sizable chunk. The breakdown of laaS market share can be seen in figure1. 


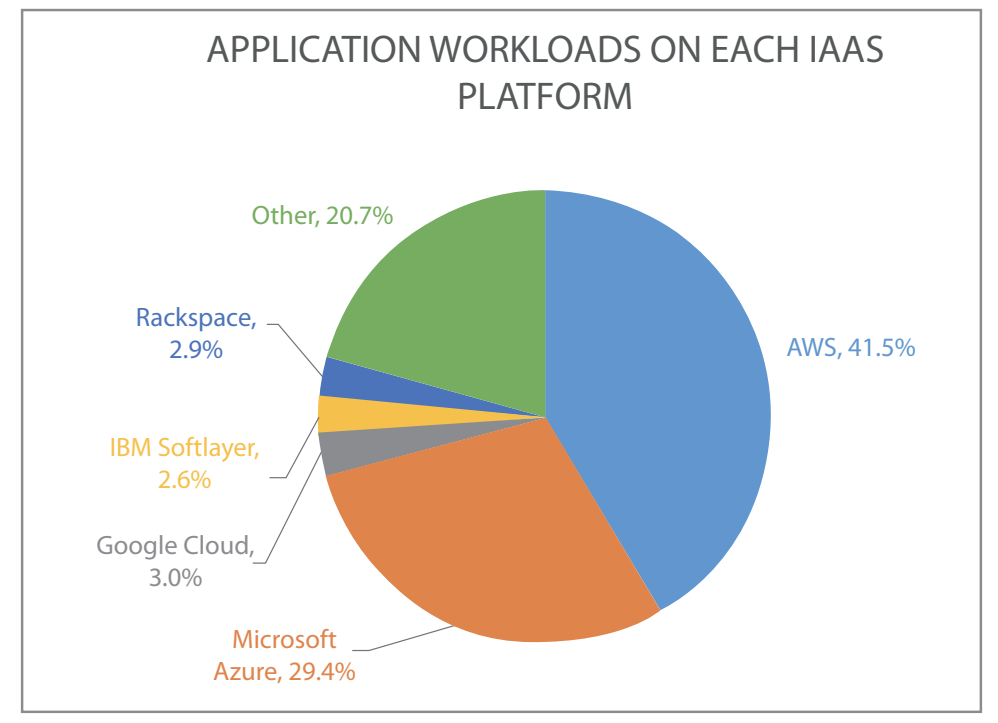

Figura 1. Cloud laaS Market Share [2].

In 2017, a majority of application workloads exe- cuted were still being processed in data centers, but itis projected that companies will begin to shift these workloads over to the public cloud in the nextone to two years. There are a number of reasons that prevent companies from adopting Cloud laaS including concerns over storing sensitive data, having their ac- count compromised by a third party user and potential misuser by end users. While these are valid concerns, $62.9 \%$ of IT professionals consider the cloud equally secure to their own data centers or even more secure in some cases leading to a more positive perspective of using cloud in the future [2]. As a result, it is important to evaluate the current cloud industry offerings in order to decide the strengths and weaknesses of each option before a company or individual jumps into the cloud industry for the first time.

\section{Usability}

In the sections to follow, analysis will be done of the three primary cloud providers regarding their current industry offerings, number of cloud-specific data centers and the best and worst aspects of their services as specified by customer reviews.

\section{Amazon Web Services}

AWS has been the dominant cloud provider since the industry first came into being. Its early launch was a large contributing factor, but its sustained market dom- inance results from its variety of service offerings and flexibility. AWS is split into the following three broad categories: Core Cloud Services, Rich Platform Ser- vices and Developer Productivity and Operational Efficiency. Each category has at least four sub-categories and the most central of the categories is the Core Cloud Services as it contains AWS's compute, storage, and networking offerings. These services are available globally thanks to AWS's nineteen data centers placed at different regions of the world (figure 2) along with the four additional data centers to be built in the near future[3].

Based off recent customer reviews [4], the most beneficial aspect of AWS is its large variety ofavailable services and the flexibility users have in leveraging them. With the large number of customers AWS ser- vices, it is vital that their product meets the different needs of their clientle in an effective manner. On the other hand, AWS's major flaw is its' lack of adequate customer 
support. While there is documentation for its various services, it can be difficult to find the topic most relevant for an issue a user is dealing with. Overall, customer reviews are quite positive when discussing AWS and on Trust Radius, reviewers gave it a 8.8/10 score on their likelihood to recommend [4].

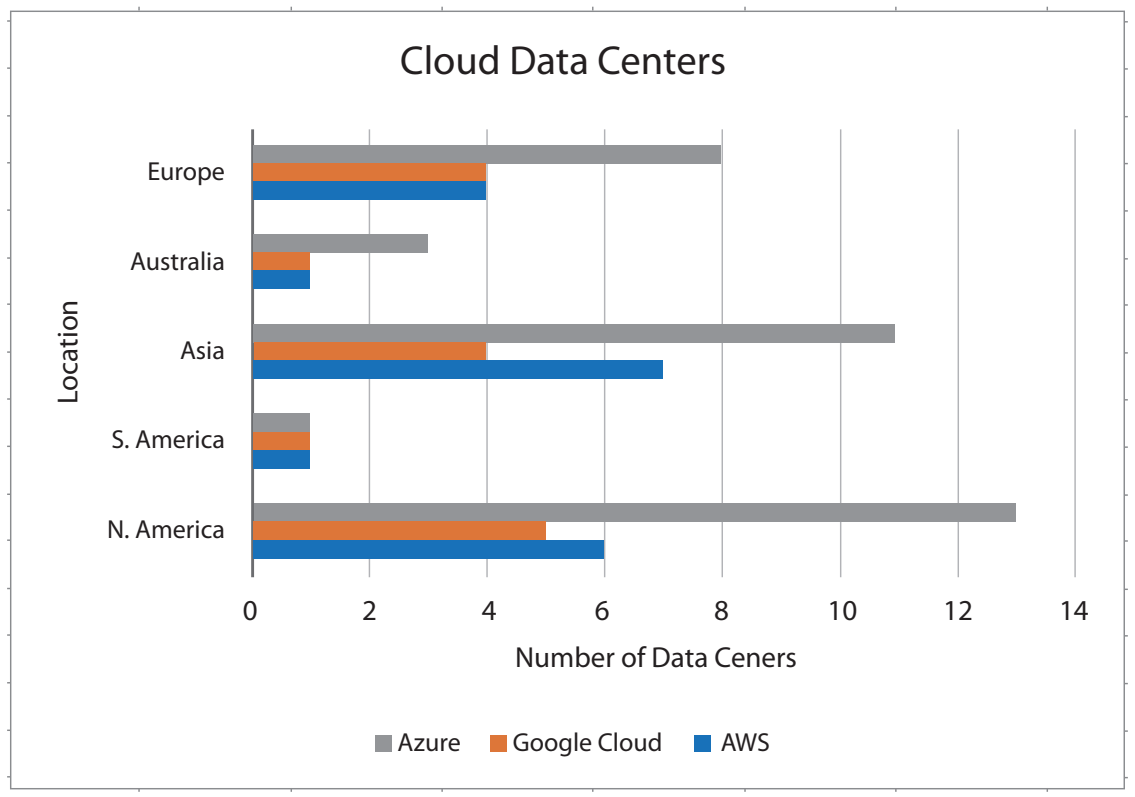

Figura. 2. Count of Cloud Data Centers in different Continents.

\section{GoogleCloud}

GC has four main categories for their available ser- vices including Computing, Storage, Networking and Management. They have fifteen data centers spread over the world for their clients.(figure 2) Even though they have fewer centers in comparison to AWS, the implementation time for their available on-demand resources is extremely fast. Over the next few years, GC has plans to build an additional four data centers. Moreover, GC boasts a user interface that is simple to use and intuitive in navigating around [5].

From customer reviews [6], the best aspect of GC is its efficiency and ease of use for development and deployment. With its appealing and simple user in- terface, developers generally enjoyed the environment. GC's ability to scale up an application provides a very appealing offering to developers. GC's main fault is its lack of training services for its available features. While the UI is easy to understand, how to utilize and leverage GC's offerings is not always straightforward. This makes it difficult to use all that GC has to offer since the training documentation and videos available are not adequate. Overall, customer reviews are pretty positive when discussing GC and on Trust Radius, reviewers gave it a 8.8/10 score on their likelihood to recommend [6].

\section{Microsoft Azure}

Azure's services are grouped under twelve different categories some of which include compute, data and storage, analytics, networking, management, and Inter- net of Things. In comparison to AWS and GC, Azure has the largest number of data centers with thirty-six spread all around the world (figure 2). This provides its clients with the ability to easily scale their business solutions 
to all parts of the globe while handling a diverse set of workloads. Azure has plans to build an additional eight data centers in the near future to further expand their reach [7].

By far, the best aspect of Azure that is mentioned in customer reviews [8] is its ability to provide on- demand infrastructure scalability on a global scale. This is particularly important for larger corporations that have an invested interest in several places around the world.An additional benefit for these large corporations is Azure's easy integration with other Microsoft Office Suite products. By contrast, Azure's weakness is evident through its outdated UI and limited customization options with its available resources and templates. While those familiar with Microsoft products usually feel comfortable with the UI, most other customers have mentioned the need to update the UI to moremod- ern standards. Finally, the flexibility of using Azure's available resources is limited since there are minimal opportunities to customize them to match the needs of different clients. Overall, customer reviews are fairly positive when discussing Azure and on Trust Radius, reviewers gave it a 8.1/10 score on their likelihood to recommend [8].

\section{Performance}

The purpose of this metric is to analyze how reliable the hardware and software used by the cloud provider is to gauge its performance in a real-life scenario. In order to conduct the tests, Phoronix Test Suite version 7.8 was used. Phoronix Test Suite is an open source fully automated platform that allows testing and benchmarking. Table I contains the instance configuration for each one of the cloud providers analyzed.

Table I. Instance configuration

\begin{tabular}{|l|l|l|}
\hline \multicolumn{1}{|c|}{ AWS } & \multicolumn{1}{|c|}{ Azure } & \multicolumn{1}{c|}{ GC } \\
\hline Intel Xeon E5-2676 v3 @ 2.40GHz & Intel Xeon E5-2673 v3 @ & Intel Xeon E5-2699 v3 @ \\
Cores: 1 & 2.39GHz & 2.30GHz \\
RAM: 1 GB & Cores: 1 & Cores: 1 \\
OS: Ubuntu 16.04 & RAM: 1 GB & RAM: 1.6 GB \\
& OS: Ubuntu 16.04 & OS: Ubuntu 16.04 \\
\hline
\end{tabular}

\section{A. RAM Speed}

Ram is an important metric because much ofthe overall performance of a computer is dependent upon theRAM.Whenabusinessisselectingacloudprovider the first thing in their mind is likely going to be speed which depends on RAM. While measuring RAM can not wholly indicate how fast a VM will run, it is a good indication. Below is a graph comparing the RAM speed of AWS, $\mathrm{GC}$, and Azure. 


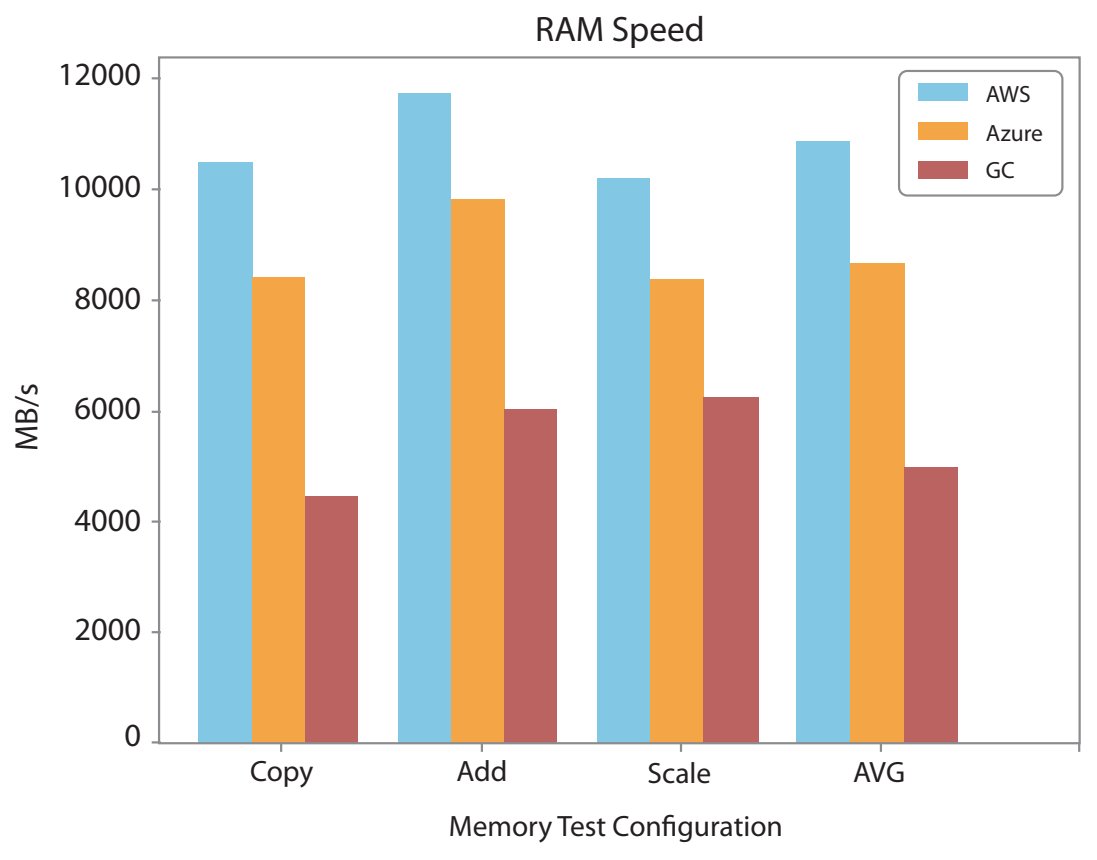

Figura 3. RAM Speed - Floating Point

As shown in figure 3 the performance of AWS is twice as better than GC. This is mainly because the hardware used by GC is not as modern as the one used by AWS (see Table I).

\section{B. Disk Performance}

The main purpose of this test is to stress the filesystem. Dbench simulates the $1 / O$ of a real server and measures the maximum workload that can be handled before the server starts lagging. This test creates, writes, reads, and deletes files of different sizes. The output of this test is the average throughput of the file system operations measured in MB/s. The following graph shows the results for Dbench test.

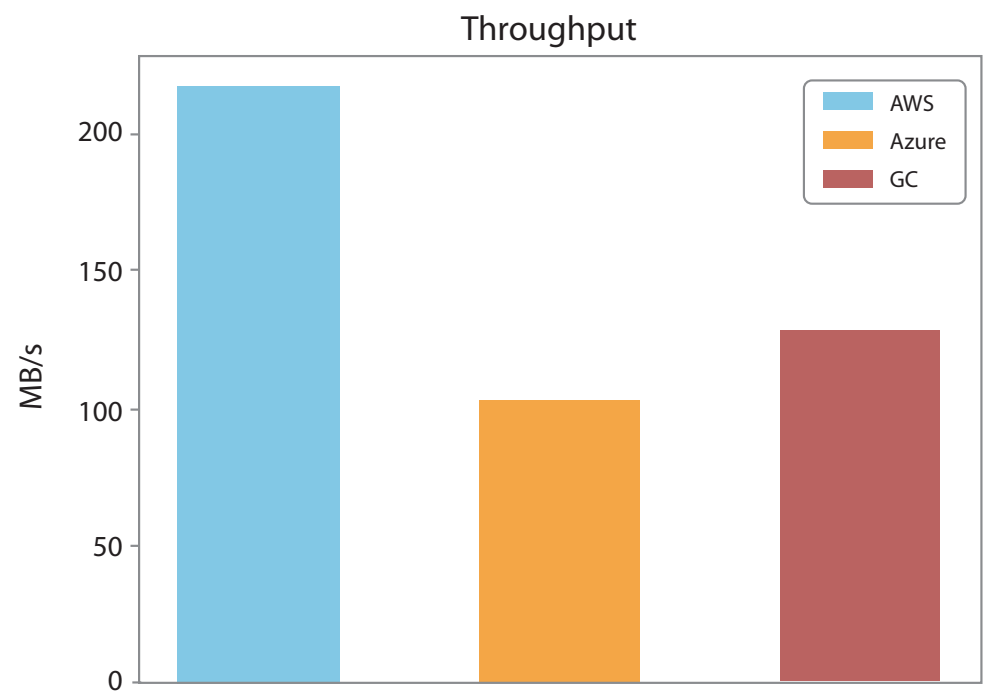

Figura 4. Disk Performance 
As can be seen, GC has a better performance than Azure. The difference between both is 20 $\mathrm{MB} / \mathrm{s}$. However, with a difference of at least $110 \mathrm{MB} / \mathrm{s}$ AWS has a significant advantage over Azure and GC again.

\section{Apache}

The Apache test measures how many requests a server can respond when having 1,000,000 requestsand 100 of them are concurrent. The test result can be seen in figure 5.

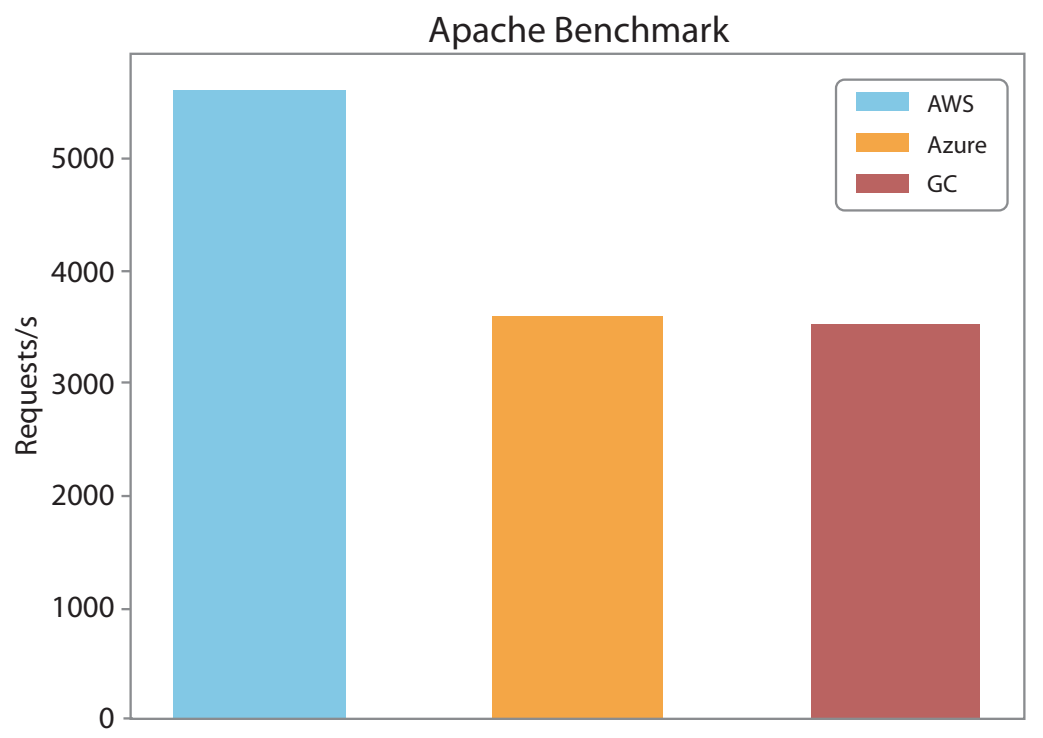

Figura 5. Apache Results

The results showed that AWS can handle an average of 5600 requests per second, while Azure and GC can handle at most 3600 requests per second. This result is valuable for the cloud users if they intend to host a web server or website using one of these providers.

\section{Cost}

We were interested in the cost metrics of the various cloud computing providers so that we could compare the cost against the usability and performance of the available cloud services. As this project focused on laaS, we narrowed the cost analysis down to virtual machine, database, and storage rentals. Below are the charts displaying our findings. 


\section{A. Virtual Machine Rental}

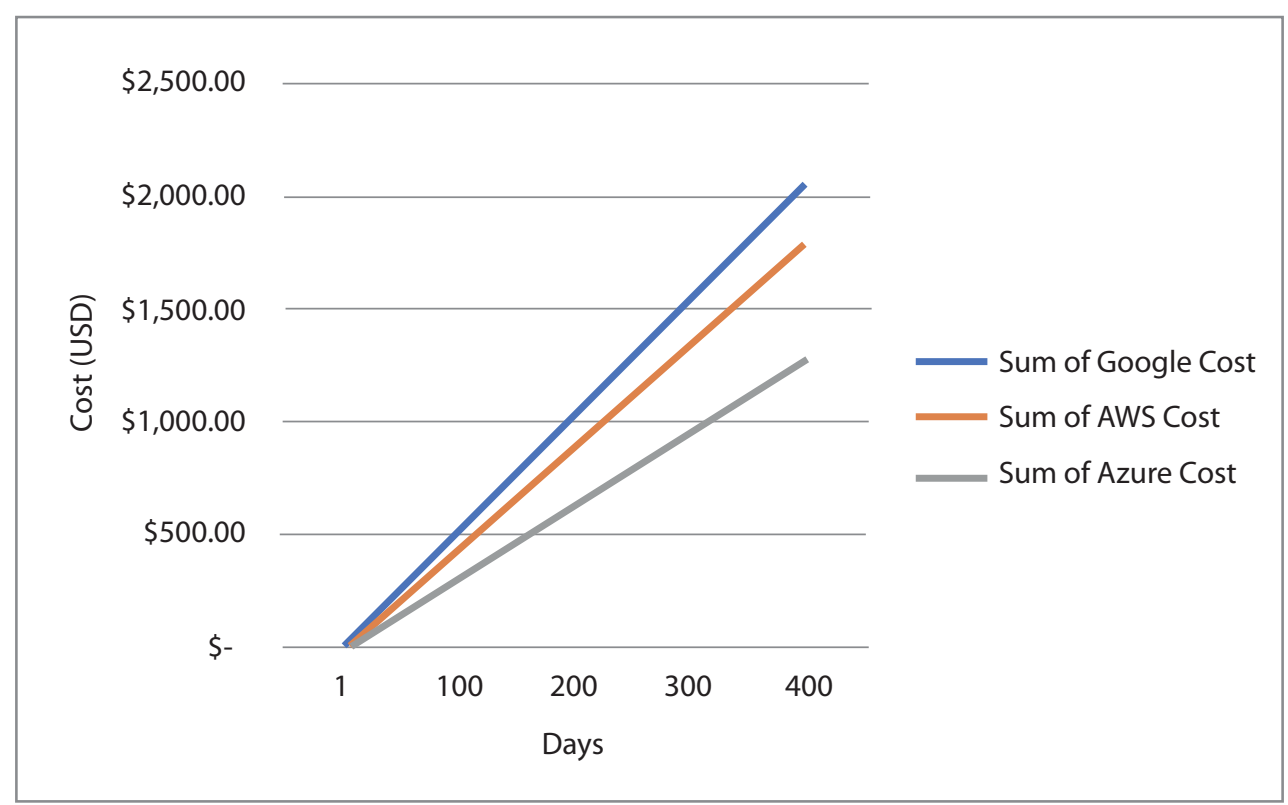

Figura 6. 15G RAM Virtual Machines

In figure 6 we examined the total cost of running a UNIX instance with 15G Ram over a one year span. We chose this benchmark since this offering would commonly be used by medium to large companies and research facilities. As time goes on Azure continues to separate itself from the competition. The graph then demonstrates Azure's clear favoritism towards larger corporations as alluded to earlier in the usability portion.

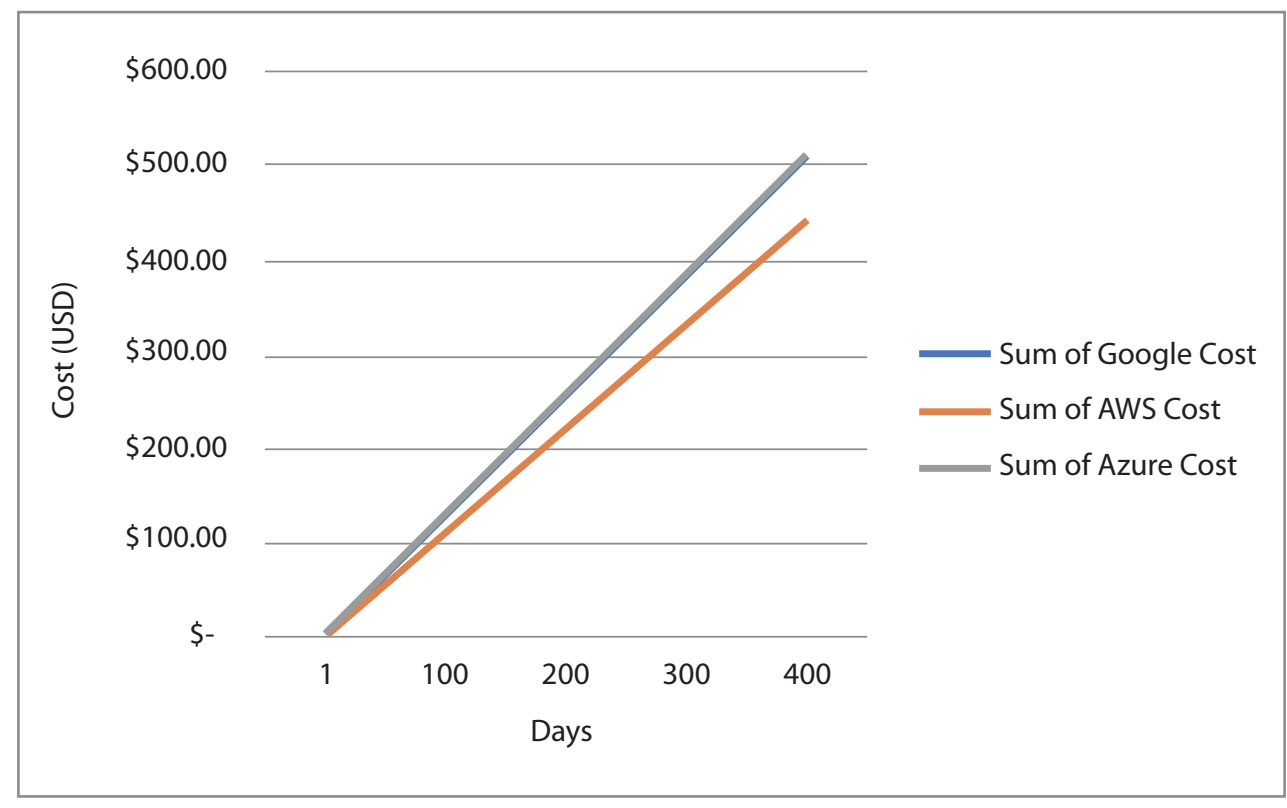

Figura 7. 4G RAM Virtual Machines 
As shown in figure 7 we examined what the cost of running a UNIX instance with 4G RAM over a year span. We chose this benchmark ( $4 G$ ) to serve as a contrast to the $15 \mathrm{G}$ chart pictured in figure 6 . The reason being is that while a $15 \mathrm{G}$ machine would serve large enterprises a $4 \mathrm{G}$ machine would serve smaller business to the everyday user. AWS is far in front of the other VMs in this category as it not only provides better hardware, it is also offered at a lower cost. One consideration, however, is that for the smaller user Google offers much more free credit to play around with than the other two options.

\section{B. Storage Rental}

The cost of storage in various cloud providers is a very important metric to consider as many other services often depend upon data stored in these services

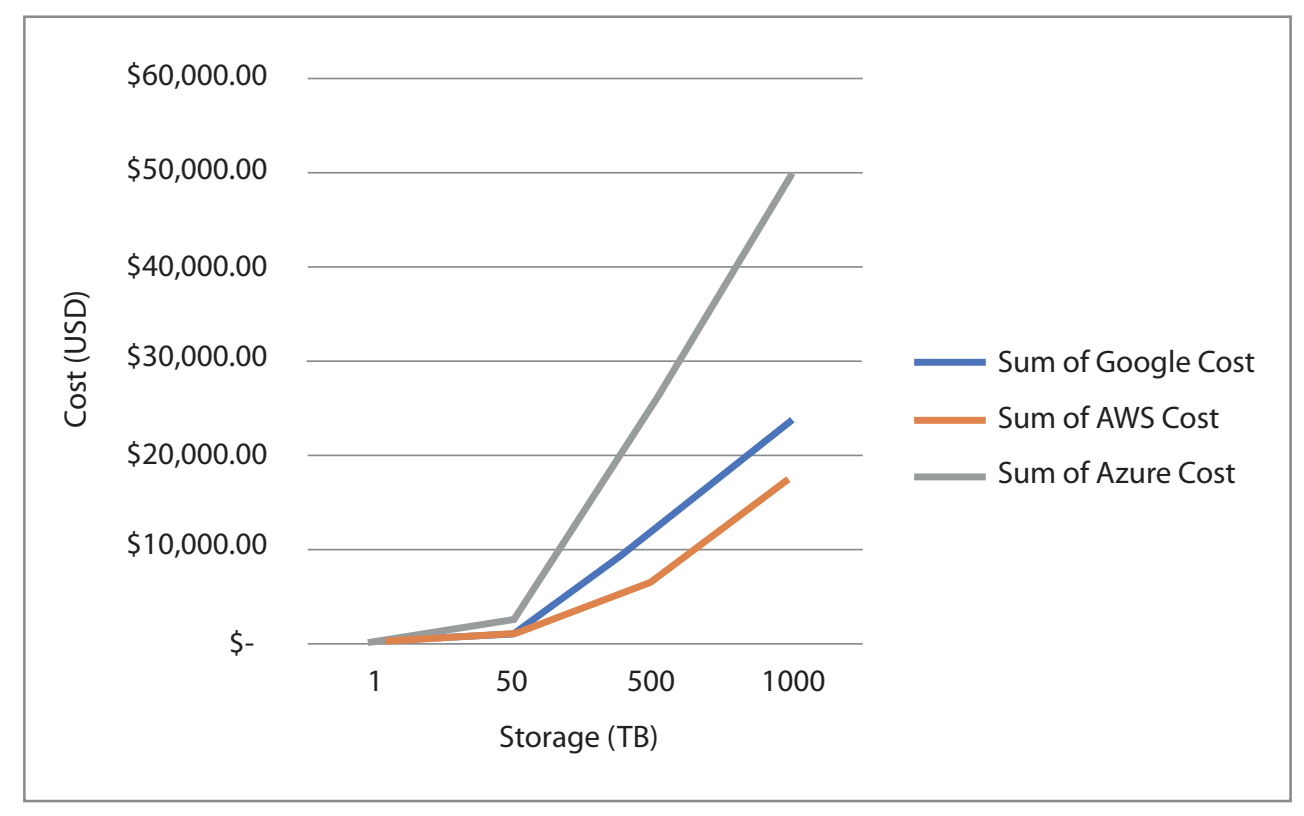

Figura 8. Storage

In the case of figure 8 above we examined the cost of storing 1TB-1000TB of data in each of therespective cloud providers. The scale of the $\mathrm{x}$-axis is not constant because both AWS and Azure have varying levels of cost per GB depending on how much data is stored (1-50, 50-500, 500-), while Google's cost is constant. The graph shows us that while all providers are closein cost for small projects, as you scale up AWS becomes continuously cheaper.

\section{Relational Database Rental}

This metric too is very important when consid- ering the best overall cloud provider because many other services depend upon information stored in these databases. For the below figures the cost is calculated using a Microsoft SQL Server because of its' popularity. 


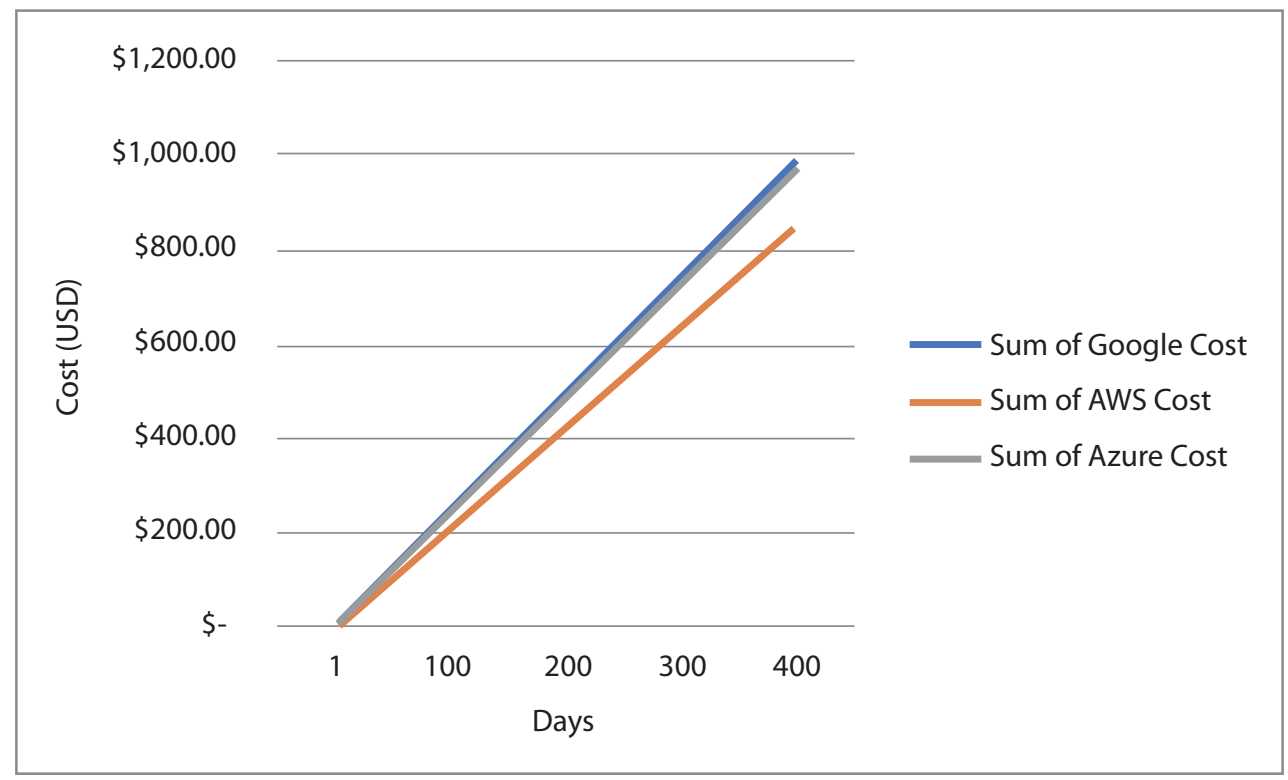

Figura 9. Medium Database

In figure 9 we compared the cost of renting a medium sized database (exact metrics differ slightly between cloud providers) on AWS, GC, and Azure. Just like the 15G Ram instances, medium to large companies are the target audience of this size database. Google Cloud and Azure's cost difference becomes negligible as time goes on, but AWS continues to be the cheapest option.

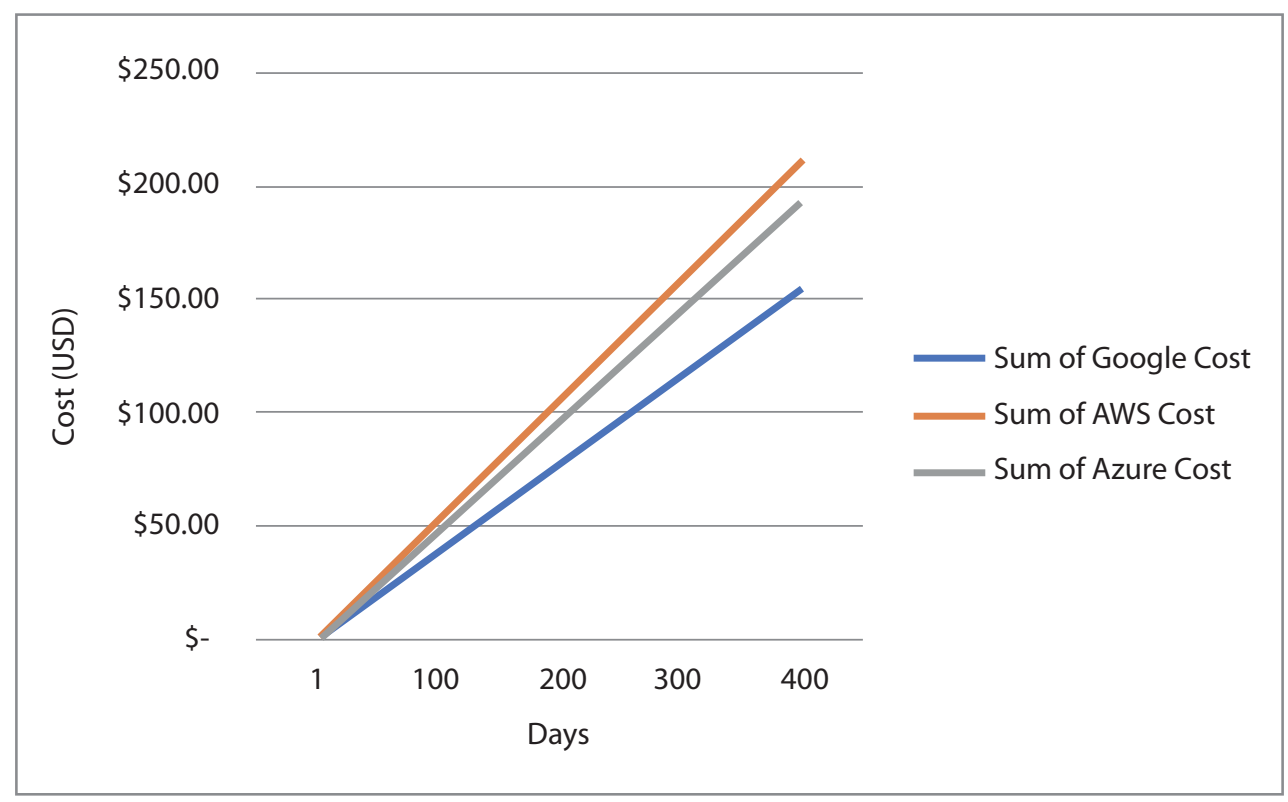

Figura 10. Micro Database

Figure 10 shows the cost comparison of rentinga micro sized database (exact metrics differ slightlybetween cloud providers) on AWS, GC, and Azure. This sized database is geared towards those doing apersonal project, or a small sized company. Here wesee a change in best option from the larger database asGC emerges as a cheap alternative to AWS or Azure. 


\section{Conclusions \& Future Work}

Each of the cloud providers have their strengths and weaknesses as presented in this paper. While this is not a comprehensive analysis of each of the providers, by selecting the three most prominent characteristics, usability, performance, and cost, we were able to get a deep understanding of each of the studied providers.

After evaluating the results of this research, AWS became the clear winner for the best overall cloud provider. With AWS's wide array of features, impressive hardware, and reasonable pricing, it is the best overall option for businesses and individuals seeking to leverage the power of cloud computing. While GC and Azure are not able to meet this same level of excellence, GC is still a decent cloud offering specifically for small groups and individuals and Azure is a good option for large corporations and businesses. The above conclusion is supported by the fact that AWS has not only the largest, but a growing market share.

To expand upon this work, it would be beneficial to add other cloud providers, notably IBM SmartCloud and Rackspace. Adding more providers would create a more holistic view of the cloud market since the ones covered in this paper only account for approximately seventy percent of the laaS market. Additionally, by including analysis of the PaaS and SaaS markets, a better understanding of the cloud computing industry as a whole can be obtained. The analysis of these other markets is important since many customers often use more than just laaS with their cloud providers. Lastly, our performance tests only addressed a small part of the overall performance of the cloud services offered. Additional tests may include: boot-times, CPU speeds, and penetration testing.

\section{Acknowledgment}

Thank you to Dr. Sai Mukkavilli for the resources required to do this project.

\section{References}

[1] "Global Cloud Computing", MarketLine. London, UK. De- cember2017.

[2] C. Coles, "AWS vs Azure vs Google Cloud Market Share 2017", Skyhigh.2017

[3] S. Vaughan-Nichols, "Amazon Web Services", PC Mag. November2016.

[4] "Amazon Web Services Reviews", TrustRadius.

[5] S. Vaughan-Nichols, "Google Cloud Platform", PC Mag. December2015.

[16] "GoogleComputeEngineReviews", TrustRadius.

[7] S. Vaughan-Nichols, "Microsoft Azure", PC Mag. July2017.

[8] "Microsoft Azure Reviews", TrustRadius.

[9] Qi Zhang, Lu Cheng, Raouf Boutaba. Cloud computing: state- of-the-art and research challenges. The Brazilian Computer Society,2010.

[10] Brian Hayes. 'Cloud computing'. In: Commun. ACM 51.7 (2008), pp. 911. ISSN: 0001-0782. url: http://portal. acm.org/citation.cfm?doid=1364782.1364786.

[11] Keqiang He, Alexis Fisher, Liang Wang, Aaron Gember, Aditya Akella, Thomas Ristenpart. Next Stop, the Cloud: Understanding Modern Web Service Deployment in EC2 and Azure. Isbn: 978-1-4799-6191-7. IEEE,2014.

[12] Rabi Prasad Padhy, Manas Ranjan Patra, Suresh Chandra Satapathy. Cloud Computing: Security Issues and Research Challenges. ISSN: 2249-9555. IRACST, Dec 2011.

[13] Phoronix Test Suite. Available: https://www.phoronix-test- suite.com 